\title{
Fasting glucose level and all-cause or cause-specific mortality in Korean adults: a nationwide cohort study
}

\author{
Yi-Suk Kim, Yong-Moon Park², Kyung-Do Han², Jae-Seung Yun ${ }^{4}$, Yu-Bae Ahn, and Seung-Hyun $\mathrm{Ko}^{4}$
}

\begin{abstract}
${ }^{1}$ Department of Anatomy Catholic Institute for Applied Anatomy, The Catholic University of Korea, Seoul, Korea; ${ }^{2}$ Epidemiology Branch, National Institute of Environmental Health Sciences, National Institutes of Health, Durham, NC, USA; ${ }^{3}$ Department of Biostatistics, College of Medicine, The Catholic University of Korea, Seoul; ${ }^{4}$ Division of Endocrinology and Metabolism, Department of Internal Medicine, St. Vincent's Hospital, College of Medicine, The Catholic University of Korea, Seoul, Korea
\end{abstract}

Received: October 26, 2019 Revised : December 26, 2019 Accepted: January 14, 2020

\section{Correspondence to}

Seung-Hyun Ko, M.D.

Division of Endocrinology and Metabolism, Department of Internal Medicine, St. Vincent's Hospital, College of Medicine, The Catholic University of Korea, 93 Jungbu-daero, Paldal-gu,

Suwon 16247, Korea

Tel: +82-31-249-8155

Fax: +82-31-253-8898

E-mail: kosh@catholic.ac.kr https://orcid.org/0000-00033703-1479
Background/Aims: Although people with diabetes have been shown to have higher mortality than people without diabetes, there is a lack of data on the association between fasting glucose (FG) levels and cause-specific mortality rates in the general population.

Methods: A total of 326,547 Korean adults over 20 years of age, who had received a health checkup between 2006 and 2008 were selected from the Korean National Health Insurance Service sample cohort dataset and followed until 2015. We estimated hazard ratios (HRs) of all-cause mortality and cause-specific mortality relative to various range of FG levels. All causes of death were classified according to International Classification of Diseases (ICD)-10 codes.

Results: During follow-up (mean, 8.5 years), a total of 13,536 deaths (mortality rate 4.89/1,000 person-year) occurred; 4,916 deaths from cancer, 2,133 from cardiovascular disease, 762 from infectious disease, 199 from renal disease, and 5,526 from other causes. The overall mortality rate increased with an increase in FG category (HR, 1.78; 95\% confidence interval, 1.65 to 1.92 ; in the $\geq 160 \mathrm{mg} / \mathrm{dL}$ ). In addition, a J-shaped associations was found between FG levels and all-cause mortality after adjustment for age, sex, smoking, drinking, physical activity, body mass index, diabetes mellitus medication, hypertension, and dyslipidemia. In particular, the risk of cancer-mortality with high FG levels was increased for men but not women.

Conclusions: The risk of all-cause and cause-specific mortality showed the tendency to increase when the FG level was outside of the normal range, indicating a J-shaped relationship, in both men and women.

Keywords: Fasting; Blood glucose; Mortality; Diabetes mellitus; Cohort study

\section{INTRODUCTION}

Glycemic control involves maintaining a normal level of blood glucose and is of vital strategic importance to prevent the risk of new-onset diabetes mellitus (DM) [1-3].
For this reason, fasting glucose (FG) is a useful indicator for screening and diagnosing diabetes mellitus. Additionally, it has recently been reported that an increased risk of mortality was associated with FG levels in a population-based study [4-6]. According to the literature, low 
or high levels of FG have been linked with an increased risk of all-cause and cause-specific mortality, indicating a J- or U-shaped relationship between FG and mortality [4,7-9]. However, existing studies have been limited in that they have been mainly focused on the risk of cardiovascular mortality [6-8], and when classifying cause-specific mortality, the causes of death were not adequately diverse, improper categories for all-cause and cause-specific mortality were defined, or unnatural deaths were included $[4-6,9,10]$.

The risks of all-cause and cause-specific mortality vary according to sex, race, and the region in which people live $[4,11]$. Recent findings have suggested ethnicity-dependent responses to insulin sensitivity, $\beta$-cell function, physical exercise, and lifestyle, which may lead to inconsistent outcomes [12-14]. It is also known that mean FG levels differ by sex and age across ethnic and regional groups $[9,15]$. Moreover, just as mortality rates differ across sexes and age groups $[16,17]$, the association of FG levels with mortality may also vary significantly, depending on sex and age.

Therefore, to address these gaps in the existing literature, we examined the association between FG and cause-specific mortality in the general population to elucidate whether the association varies by sex and age in Korean adults by using a retrospective cohort study design utilizing the National Sample Cohort (2002 to 2015) database released from the Korean National Health Insurance Service (NHIS).

\section{METHODS}

\section{Study population}

The NHIS was initiated in 1963 in Korea as part of the National Health Insurance Act, and it is mandatory for Korean citizens to participate in this health policy. In the present study, we used data from the National Sample Cohort 2.0 (2002 to 2015) database (NHIS-NSC) released from the Korean NHIS; the data comprise a nationally representative random sample of 1,025,340 individuals (approximately $2.2 \%$ of the entire population) established the initial cohort in 2002 and were followed for 14 years to 2015. The data were built by using probabilistic sampling to represent an individual's total annual medical expenses within each of 1,476 strata defined by age, sex, eligibility status (employed or self-employed), and income level (20 quantiles for each eligibility status and medical-aid beneficiary) combinations [18]. The size and scale of the NHIS-NSC database has been detailed in other publications $[19,20]$. The NHIS-NSC is a semi-dynamically constructed cohort database; the cohort was followed to either the time of the participant's disqualification from receiving health services due to death or emigration or until the end of the study period, and newborn infants entered the cohort annually. The database contains eligibility and demographic information regarding health insurance as well as data on medical aid beneficiaries, medical billing details, medical treatments, disease histories and prescriptions; these data were constructed after converting insurance claim information to the first day of medical treatment.

From this cohort, we selected 326,547 Korean adults over 20 years of age who had received a health checkup between 2006 and 2008. This cohort was followed from the index date until the end of the study period (i.e., December 31, 2015) or the last year of qualification for those who were alive, or until the date of death for those who died. We excluded 26,547 individuals who were missing data for at least one of the following clinical parameters: body mass index (BMI), systolic blood pressure (SBP), diastolic blood pressure (DBP), glucose, total cholesterol, hypertension, dyslipidemia or self-reported smoking, drinking habits, or physical exercise. This study was approved by the NHIS inquiry commission. The personal privacy of each participant was protected by deidentification of the national insurance claims data for analysis. This study was also approved by the Institutional Review Board of St. Vincent's Hospital (No. VC18ZESIo260). Written informed consent by the patients was waived due to a retrospective nature of our study.

\section{Measurements and definitions}

The FG level was obtained from laboratory data during regular medical check-up programs provided by the NHIS. For most participants, their serum glucose level was measured after fasting for at least 8 hours. The FG level at baseline was categorized into seven groups from lowest $(<70 \mathrm{mg} / \mathrm{dL}$ ) to highest $(\geq 160 \mathrm{mg} / \mathrm{dL}$ ) and incremented by $10 \mathrm{mg} / \mathrm{dL}$. The remaining groups were 70 to 79, 80 to 99,100 to 125,126 to 139 , and 140 to $159 \mathrm{mg} / \mathrm{dL}$. 
In this study, DM was defined as $\mathrm{FG} \geq 126 \mathrm{mg} / \mathrm{dL}$ as recommended by the 2017 American Diabetes Association guidelines [21] or treatment history with hypoglycemic medication (insulin or oral hypoglycemic agents) and/or a self-reported history of DM.

In the sub-cohort for sensitivity analysis that consisted of the participants who received regular medical check-ups, information concerning smoking status (never smoked; former smoker; current smoker), drinking habit (never drank alcohol; moderate drinker, $\leq 1$ drink per day; heavy drinker, >1 drink per day), and physical activity (no exercise, 1 to 2 times per week, 3 to 4 times per week, 5 to 6 times per week, everyday) was obtained using a standard questionnaire during the health examination. BMI was calculated as weight in kilograms divided by the square of height in meters (kg/ $\mathrm{m}^{2}$ ). Hypertension was defined by claim codes (International Classification of Diseases [ICD]-10, I10-I13, and I15 and prescription of anti-hypertensive agents or systolic or diastolic blood pressure $\geq 140$ and $\geq 90 \mathrm{mmHg}$ ). Dyslipidemia was defined by claim codes (ICD-10, E78, and prescription of lipid lowering agents or total cholester$\mathrm{ol} \geq 6.2 \mathrm{mmol} / \mathrm{L}$ ). The 1oth revision of the ICD-10 codes for diabetes, including E1o (insulin-dependent diabetes mellitus), E11 (non-insulin-dependent diabetes mellitus), E12 (malnutrition-related diabetes mellitus), E13 (other specified diabetes mellitus), or E14 (unspecified diabetes mellitus), were assigned as either the principal or additional diagnosis. Antidiabetic drugs dispensed by the pharmacy in Korea during the study period consisted of six classes (i.e., sulfonylureas, biguanide, alpha-glucosidase inhibitor, thiazolidinediones, meglitinide and insulin).

\section{Mortality data and causes of death}

Information on mortality and cause of death was available for all subjects in the cohort; the latter was classified according to the Korean Standard Classification of Diseases and Causes of Death [22], based on the ICD-10, as provided by the Korean National Statistical Office. Any cases of accidental death were excluded. Diseases or conditions directly leading to death were adopted as the specific causes of death, which were categorized according to their ICD-10 codes as follows: malignant neoplasm (Coo-97 and Doo-Do9), cardiovascular disease (CVD: Ioo-Io2, I05-I15, I20-I25, and I6o-I79), infectious disease (Aoo-B99, Joo-Jo6, Jo9-J18, J20-J22, and J85-J86), renal disease (Noo-No8 and N10-N19), and other causes (codes other than those mentioned above and excluding any unnatural deaths such as suicide and accidental death).

\section{Statistical analysis}

The association between FG level and mortality was analyzed with Cox proportional hazard models. Hazard ratios (HRs) and their 95\% confidence intervals (CIs) were calculated for each FG group, relative to the reference (FG, 80 to $99 \mathrm{mg} / \mathrm{dL}$ ) after adjusting for age, sex, BMI, smoking status, alcohol consumption, physical exercise, DM medication, hypertension, and dyslipidemia. Furthermore, to evaluate the robustness of the association between FG level and mortality, stratified analyses according to the subjects' sex and age group (20 to 39, 40 to $64, \geq 65$ years) were performed. All statistical analyses were performed using SAS software version 9.4 (SAS Institute Inc., Cary, NC, USA). All p values were two-tailed, and values less than 0.05 were considered statistically significant.

\section{RESULTS}

A total of 172,605 men and 153,942 women were eligible for inclusion in the study. Table 1 shows the baseline characteristics of participants grouped by sex and the FG categories. The mean ages of the men and women were $45.4( \pm 13.9)$ years and $47.9( \pm 15.0)$ years, respectively. Smoking, drinking, exercise, BMI, hypertension, dyslipidemia, SBP, DBP, and total cholesterol in men and women were significantly related to FG level $(p<0.001)$. BMI, SBP, DBP, glucose, total cholesterol, hypertension and dyslipidemia were linearly increased across all groups for both sexes. Additionally, no exercise and exercise 1 to 2 times per week decreased linearly across the FG groups for both sexes, and while exercise everyday increased linearly. Current smoking was increased in the individuals with fasting glucose levels $<100$ and $\geq 126 \mathrm{mg} / \mathrm{dL}$ in both sexes. Unlike the linear decrease across the FG groups observed in women, heavy drinking was not different across the FG groups in males. The characteristics of the participants with or without a history of DM medication are also shown in Table 1 . The 


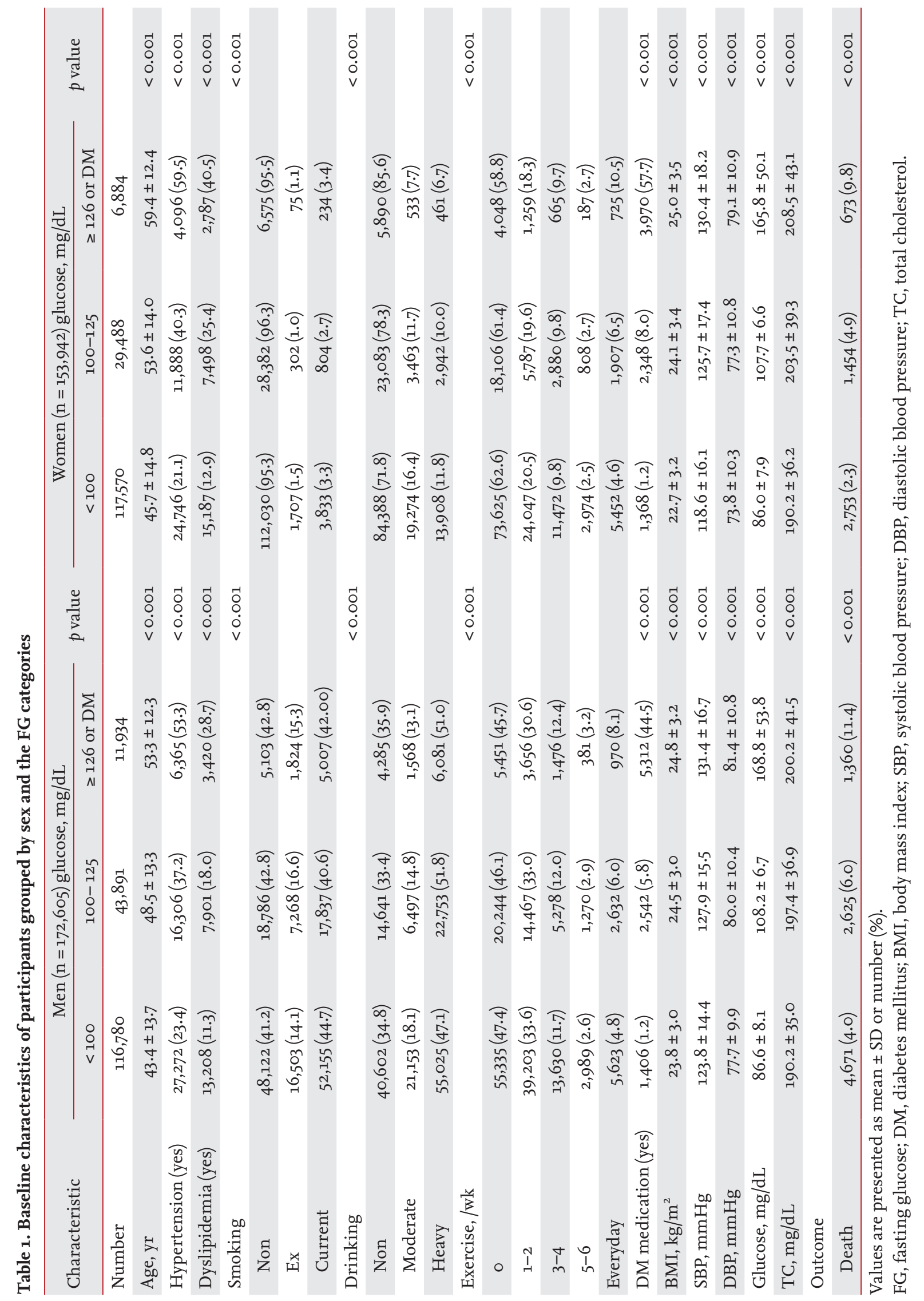


Table 2. Adjusted HRs and $95 \%$ confidence intervals for the association of fasting glucose categories with all-cause and cause-specific mortality in Korean adults

\begin{tabular}{|c|c|c|c|c|c|}
\hline Glucose, mg/dL & Number & Death & MR (per 1,000) & Crude HR & Adjusted HR \\
\hline \multicolumn{6}{|l|}{ Overall death } \\
\hline$<70$ & 3,469 & 122 & 4.11 & $1.09(0.91-1.30)$ & $1.39(1.16-1.66)^{\mathrm{a}}$ \\
\hline $70-79$ & 46,453 & 1,387 & 3.47 & $0.92(0.86-0.97)^{\mathrm{a}}$ & $1.05(0.99-1.11)$ \\
\hline $80-99$ & 184,428 & 5,915 & 3.78 & 1.00 (reference) & 1.00 (reference) \\
\hline $100-125$ & 73,379 & 4,079 & 6.62 & $1.76(1.69-1.83)^{\mathrm{a}}$ & $1.14(1.09-1.18)^{\mathrm{a}}$ \\
\hline $126-139$ & 6,581 & 557 & 10.24 & $2.73(2.50-2.97)^{\mathrm{a}}$ & $1.16(1.06-1.27)^{\mathrm{a}}$ \\
\hline $140-159$ & 4,803 & 465 & 11.69 & $3.11(2.83-3.42)^{\mathrm{a}}$ & $1.20(1.07-1.33)^{\mathrm{a}}$ \\
\hline$\geq 160$ & 7,434 & 1,011 & 16.84 & $4.5^{1}(4.22-4.82)^{\mathrm{a}}$ & $1.78(1.65-1.92)^{\mathrm{a}}$ \\
\hline \multicolumn{6}{|l|}{ Death, cancer } \\
\hline$<70$ & 3,469 & 47 & 1.59 & $1.09(0.82-1.46)$ & $1.40\left(1.05^{-1.88}\right)^{\mathrm{a}}$ \\
\hline $70-79$ & 46,453 & 496 & 1.24 & $0.85(0.78-0.94)^{a}$ & $0.98(0.89-1.08)$ \\
\hline $80-99$ & 184,428 & 2,269 & 1.45 & 1.00 (reference) & 1.00 (reference) \\
\hline $100-125$ & 73,379 & 1,511 & 2.45 & $1.70(1.59-1.81)^{\mathrm{a}}$ & $1.13(1.06-1.21)^{\mathrm{a}}$ \\
\hline $126-139$ & 6,581 & 188 & 3.46 & $2.40(2.07-2.78)^{\mathrm{a}}$ & $1.12(0.96-1.30)$ \\
\hline $140-159$ & 4,803 & 148 & 3.72 & $2.58(2.18-3.05)^{\mathrm{a}}$ & $1.11(0.93-1.32)$ \\
\hline$\geq 160$ & 7,434 & 257 & 4.28 & $2.98(2.62-3.39)^{\mathrm{a}}$ & $1.35(1.16-1.56)^{\mathrm{a}}$ \\
\hline \multicolumn{6}{|c|}{ Death, cardiovascular } \\
\hline$<70$ & 3,469 & 11 & 0.37 & $0.63(0.35-1.14)$ & $0.84(0.47-1.53)$ \\
\hline $70-79$ & 46,453 & 212 & 0.53 & $0.90(0.78-1.05)$ & $1.06(0.91-1.23)$ \\
\hline $80-99$ & 184,428 & 918 & 0.59 & 1.00 (reference) & 1.00 (reference) \\
\hline $100-125$ & 73,379 & 663 & 1.08 & $1.84(1.67-2.04)^{\mathrm{a}}$ & $1.11(1.00-1.23)$ \\
\hline $126-139$ & 6,581 & 85 & 1.56 & $2.68(2.15-3.35)^{\mathrm{a}}$ & $1.05(0.83-1.32)$ \\
\hline $140-159$ & 4,803 & 80 & 2.01 & $3.45(2.74-4.33)^{\mathrm{a}}$ & $1.22(0.96-1.56)$ \\
\hline$\geq 160$ & 7,434 & 164 & 2.73 & $4.72(3.99-5.57)^{\mathrm{a}}$ & $1.75\left(1.45^{-2.12}\right)^{\mathrm{a}}$ \\
\hline \multicolumn{6}{|l|}{ Death, infectious } \\
\hline$<70$ & 3,469 & 8 & 0.27 & $1.33(0.66-2.68)$ & $1.62(0.80-3.28)$ \\
\hline $70-79$ & 46,453 & 84 & 0.21 & $1.03(0.81-1.32)$ & $1.15(0.91-1.47)$ \\
\hline $80-99$ & 184,428 & 316 & 0.20 & 1.00 (reference) & 1.00 (reference) \\
\hline $100-125$ & 73,379 & 225 & 0.37 & $1.82(1.53-2.16)^{\mathrm{a}}$ & $1.18(1.00-1.41)$ \\
\hline $126-139$ & 6,581 & 36 & 0.66 & $3.32(2.35-4.68)^{\mathrm{a}}$ & $1.53(1.07-2.18)^{\mathrm{a}}$ \\
\hline $140-159$ & 4,803 & 30 & 0.75 & $3.77(2.60-5.49)^{\mathrm{a}}$ & $1.58(1.06-2.34)^{\mathrm{a}}$ \\
\hline$\geq 160$ & 7,434 & 63 & 1.05 & $5.30(4.05-6.95)^{\mathrm{a}}$ & $2.35(1.72-3.20)^{\mathrm{a}}$ \\
\hline \multicolumn{6}{|l|}{ Death, renal } \\
\hline$<70$ & 3,469 & 3 & 0.10 & $2.07(0.65-6.56)$ & $2.89(0.91-9.17)$ \\
\hline $70-79$ & 46,453 & 21 & 0.05 & $1.08(0.66-1.74)$ & $1.30(0.80-2.10)$ \\
\hline $80-99$ & 184,428 & 76 & 0.05 & 1.00 (reference) & 1.00 (reference) \\
\hline $100-125$ & 73,379 & 50 & 0.08 & $1.68(1.17-2.40)^{\mathrm{a}}$ & $0.86(0.60-1.24)$ \\
\hline $126-139$ & 6,581 & 12 & 0.22 & $4.58(2.49-8.41)^{\mathrm{a}}$ & $1.21(0.64-2.30)$ \\
\hline $140-159$ & 4,803 & 8 & 0.20 & $4.17(2.01-8.63)^{\mathrm{a}}$ & $0.95(0.44-2.05)$ \\
\hline$\geq 160$ & 7,434 & 29 & 0.48 & $10.09(6.58-15.47)^{\mathrm{a}}$ & $2.28(1.37-3.81)^{\mathrm{a}}$ \\
\hline \multicolumn{6}{|l|}{ Death, others } \\
\hline$<70$ & 3,469 & 53 & 1.787 & $1.20(0.91-1.57)$ & $1.90(1.13-1.96)^{\mathrm{a}}$ \\
\hline $70-79$ & 46,453 & 574 & 1.438 & $0.96(0.88-1.05)$ & $1.09(1.00-1.19)$ \\
\hline $80-99$ & 184,428 & 2,336 & 1.491 & 1.00 (reference) & 1.00 (reference) \\
\hline $100-125$ & 73,379 & 1,630 & 2.646 & $1.78(1.67-1.89)^{\mathrm{a}}$ & $1.15(1.08-1.23)^{\mathrm{a}}$ \\
\hline $126-139$ & 6,581 & 236 & 4.340 & $2.92(2.56-3.34)^{a}$ & $1.21\left(1.05^{-1.39}\right)^{\mathrm{a}}$ \\
\hline $140-159$ & 4,803 & 199 & 5.002 & $3.37(2.91-3.89)^{a}$ & $1.24(1.06-1.45)^{\mathrm{a}}$ \\
\hline$\geq 160$ & 7,434 & 498 & 8.297 & $5.61(5.10-6.18)^{\mathrm{a}}$ & $2.05(1.83-2.30)^{\mathrm{a}}$ \\
\hline
\end{tabular}

Adjusted for age, smoking, drinking, physical activity, body mass index, diabetes mellitus medication, hypertension, and dyslipidemia.

$\mathrm{HR}$, hazard ratio; MR, mortality rate.

${ }^{a} p<0.05$ vs. reference range (8o to $99 \mathrm{mg} / \mathrm{dL}$ ). 
numbers of participants with DM medication among the total population were $9,260(5.4 \%)$ in men and 7,686 (5.0\%) in women and were substantially higher in individuals with fasting glucose levels $\geq 126 \mathrm{mg} / \mathrm{dL}$ in both sexes.

During the follow-up period of 8.5 years, a total of 13,536 deaths (mortality rate 4.89/1,000 person-year) occurred; 4,916 (1.51\%) deaths from cancer, 2,133 (0.65\%) from CVD, 762 (0.23\%) from infectious disease, 199 (0.06\%) from renal disease, and 5,526 (1.69\%) from other natural causes of death. Compared with the reference group (FG 80 to $99 \mathrm{mg} / \mathrm{dL})$, the overall mortality rate increased with an increase in FG category (HR, 1.78; 95\% CI, 1.65 to 1.92, in the $\geq 160 \mathrm{mg} / \mathrm{dL}$ category, $p<0.001$ ); various cause-specific mortality rates were increased with an increase in FG category as well. The HR of other causes of natural deaths was approximately twice as high at low and high ranges of FG levels (FG, < 70 and $\geq 126 \mathrm{mg} / \mathrm{dL}$ ) (Table 2).

In the multivariable HR analyses (Fig. 1), J-shaped associations were found in men and women between FG levels and all-cause mortality after adjustment for age, sex, smoking, drinking, physical activity, BMI, DM medication, hypertension, and dyslipidemia. For both sexes, similar patterns were found in infectious disease and other causes of natural death; however, this pattern was not observed for CVD-related deaths. In deaths related to renal disease, a slight J shaped curve was shown for both sexes, although the CI of FG was not statistically significant. Furthermore, for deaths related to cancer, the mortality pattern was different between the sexes, and J-shaped associations were more prominent in men than in women.

In participants with DM, the multivariable-adjusted HRs were significantly changed at FG levels $<70 \mathrm{mg} /$ $\mathrm{dL}$ or $\geq 160 \mathrm{mg} / \mathrm{dL}$. The multivariable-adjusted HRs for the FG levels from $70-80$ to $140-160 \mathrm{mg} / \mathrm{dL}$ were not significantly changed. However, in participants without $\mathrm{DM}$, the multivariable-adjusted HRs steadily increased for FG levels $<70$ or $\geq 100 \mathrm{mg} / \mathrm{dL}$. In contrast, in the case of cardiovascular death, the multivariable-adjusted HR was significantly decreased at FG levels $<70 \mathrm{mg} / \mathrm{dL}$ regardless of DM status (Fig. 2).

Table 3 shows the HRs for all-cause mortality by FG levels according to age group, smoking status, obesity, and CVD. The J-shape pattern between FG levels and mortality was observed in participants older than 40 .
These relationships between FG levels and mortality were not changed by smoking, obesity, or CVD status. Finally, adults between 20 and 65 years old with high FG levels had a higher HR for all-cause mortality than those in the older age group (age $\geq 65$ ). The HRs of low FG levels were higher in adults over the age of 40 than in younger adults $(20 \leq$ age $<40)$.

\section{DISCUSSION}

In this Nationwide population-based cohort study, we found that the risk of all-cause natural mortality was increased when the FG level was outside of the normal range, indicating a J-shaped relationship, in both men and women. However, the risk of cancer-mortality with high FG was increased for men but not women.

Previous studies investigating associations between FG and the risk of all-cause mortality have reported J-shaped relationships, and our findings are in agreement with the cohort studies in earlier literature $[4,23,24]$. In our results, we noted this pattern in various categories of cause-specific mortality, in men and women, except in the case of cardiovascular deaths (Fig. 1). Moreover, adults between 20 and 65 years old had a larger increase in mortality with increasing FG levels (Table 3). Hence, our data support the conclusion that younger individuals who are obese and have FG levels beyond the normal range need to be more committed to managing and preventing DM.

On the other hand, it is well known that a FG level of $<70 \mathrm{mmol} / \mathrm{L}$ is associated with an increased risk of allcause mortality compared with a normal baseline fasting glucose level $[4,25]$. We also found that the risk for all-cause mortality was higher in participants with low FG levels, especially in individuals taking DM medications (Figs. 1 and 2). Although the reason for the low concentrations of glucose in the low FG population was not established (i.e., whether they had hyperinsulinemia, insulin resistance, poor nutrition or liver dysfunction) [25], the treatment strategies need to be well established to prevent fasting hypoglycemia in the group of individuals at high-risk for DM.

The significance of the risk of CVD mortality with FG in the lower range is very controversial. Positive associations for increased risk of coronary heart disease 

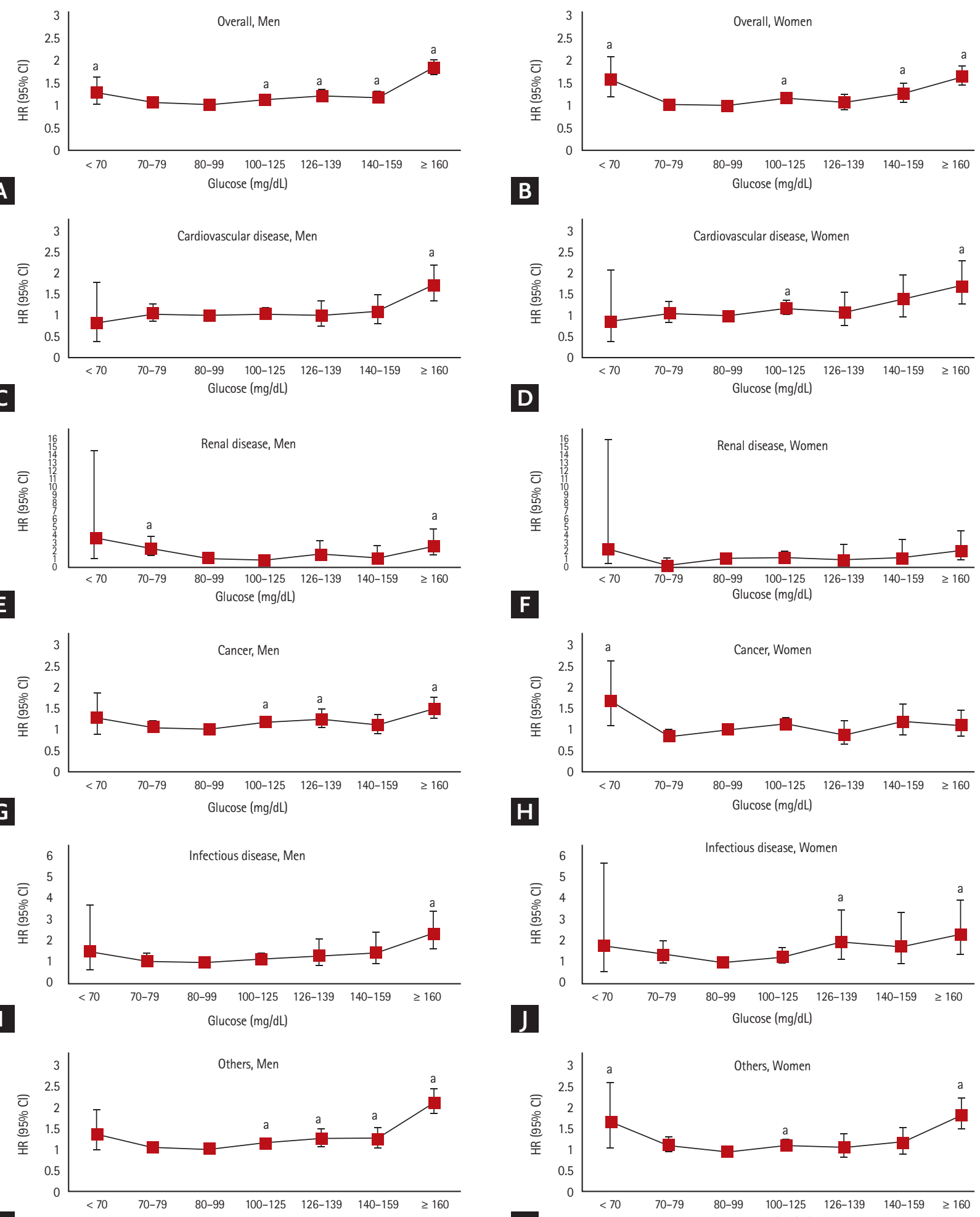

K

Glucose $(\mathrm{mg} / \mathrm{dL})$

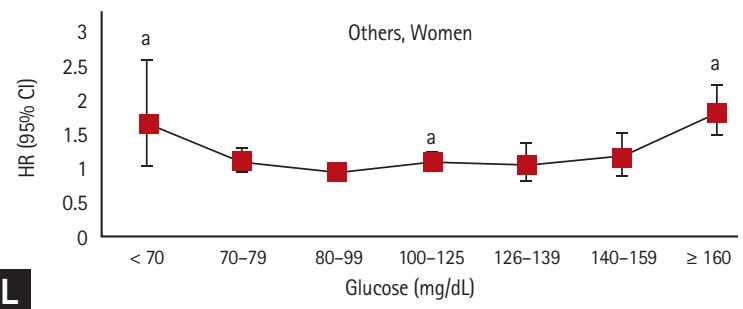

Figure 1. Adjusted Cox proportional hazard models with $95 \%$ confidence intervals (CIs) for the association of fasting glucose (FG) categories and cause-specific mortality in men (A, C, E, G, I, and K) and women (B, D, F, H, J, and L). Adjusted for age, smoking, drinking, physical activity, body mass index, diabetes mellitus medication, hypertension, and dyslipidemia. HR, hazard ratio. ${ }^{\mathrm{a}} \mathrm{p}<0.05 \mathrm{vs}$. reference range (80 to $99 \mathrm{mg} / \mathrm{dL}$ ). 


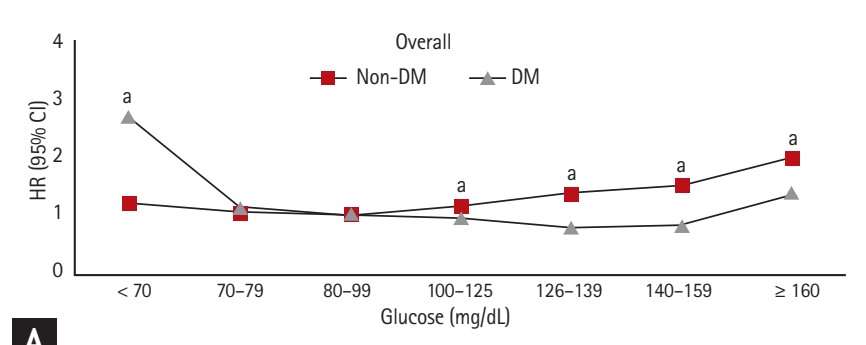

A

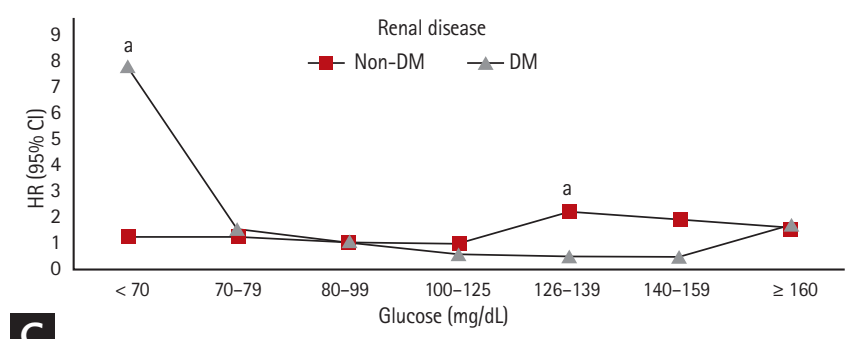

C

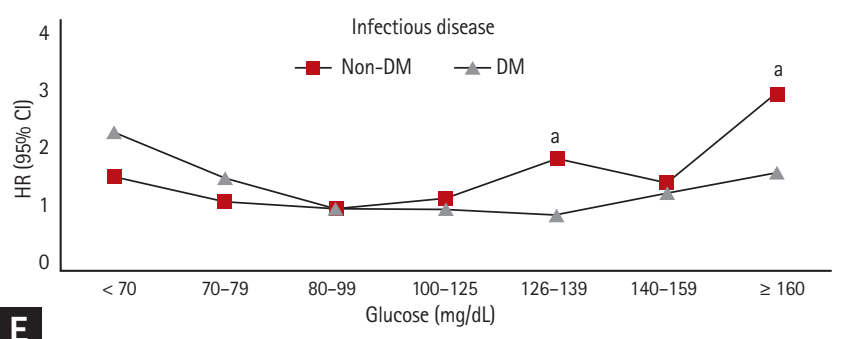

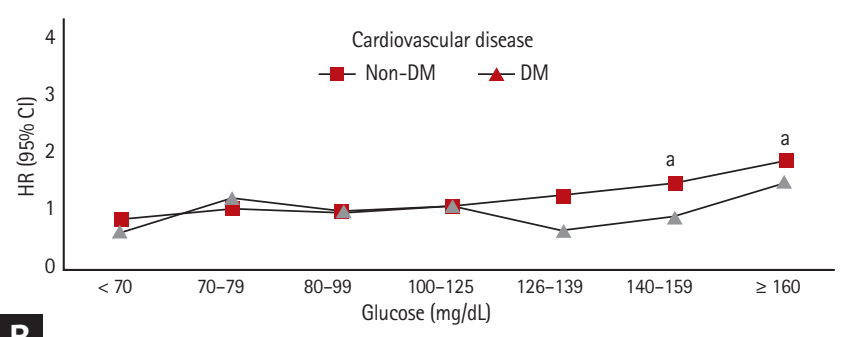

B

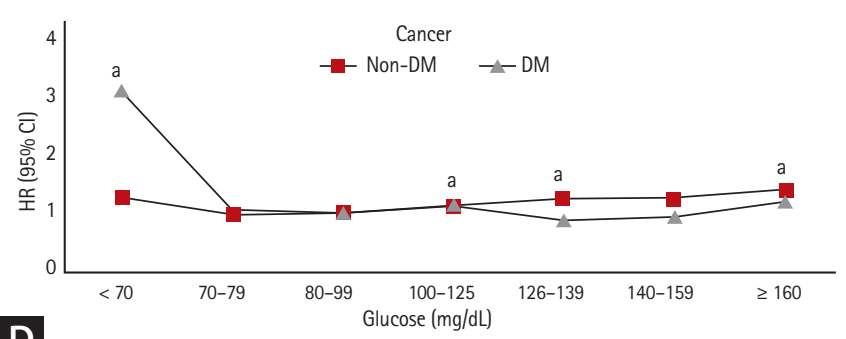

D

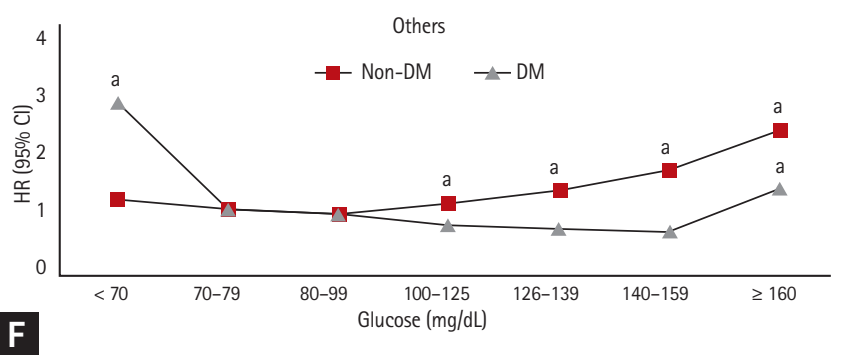

Figure 2. Adjusted Cox proportional hazard models with $95 \%$ confidence intervals for the association of fasting glucose (FG) categories and diabetes mellitus (DM) with cause-specific mortality in Korean adults. FG levels of non-DM (solid line with squares) and DM (solid line with triangles) are displayed in line graphs (A-F). Adjusted for age, sex, smoking, drinking, physical activity, body mass index, hypertension, and dyslipidemia. ${ }^{a} p<0.05$ vs. reference range (80 to $99 \mathrm{mg} / \mathrm{dL}$ ).

and stroke mortality at the low FG level have been reported by a meta-analysis and prospective cohort studies [4,7,26-28]. On the other hand, no significant associations between low FG and CVD risk or death were observed in white men from the West of Scotland Coronary Prevention Study [29] and black or white adults from the Atherosclerosis Risk in Communities (ARIC) study [30]. Some previous studies also demonstrated that FG in the lower normal range was not significantly associated with cardiovascular death in the nondiabetic healthy population [31] or in postmenopausal women [8]. These null findings were consistent with our results of the association between low FG and CVD cause-specific mortality in both men and women (Fig. 1). The possible reasons are described in the WOSCOPS study [29] for a direct role of plasma glucose as a pathogenic mediator in affecting blood vessels, such as inhibition of smooth muscle cell apoptosis, stimulation of inflammation and oxidative stress, low-density lipoprotein oxidation and increasing thrombotic potential. Although variations in the source population or analyses, including adjustment for diabetes medication use, may explain these differences, they should be considered when analyzing mortality data and classifying cause of death. Therefore, further research and perhaps pooled analyses of data from large studies should be performed using flexible models to confirm our findings of an association between the lower normal range of FG levels and low CVD mortality.

To our knowledge, this is the first study to explore the association of cancer mortality with FG levels by sex. In this study, the HRs for cancer mortality with FG levels $\geq$ $100 \mathrm{mg} / \mathrm{dL}$ were different by sex; the risk of cancer-mortality with high FG levels was increased for men but not women (Fig. 1). The mechanism of increased cancer 


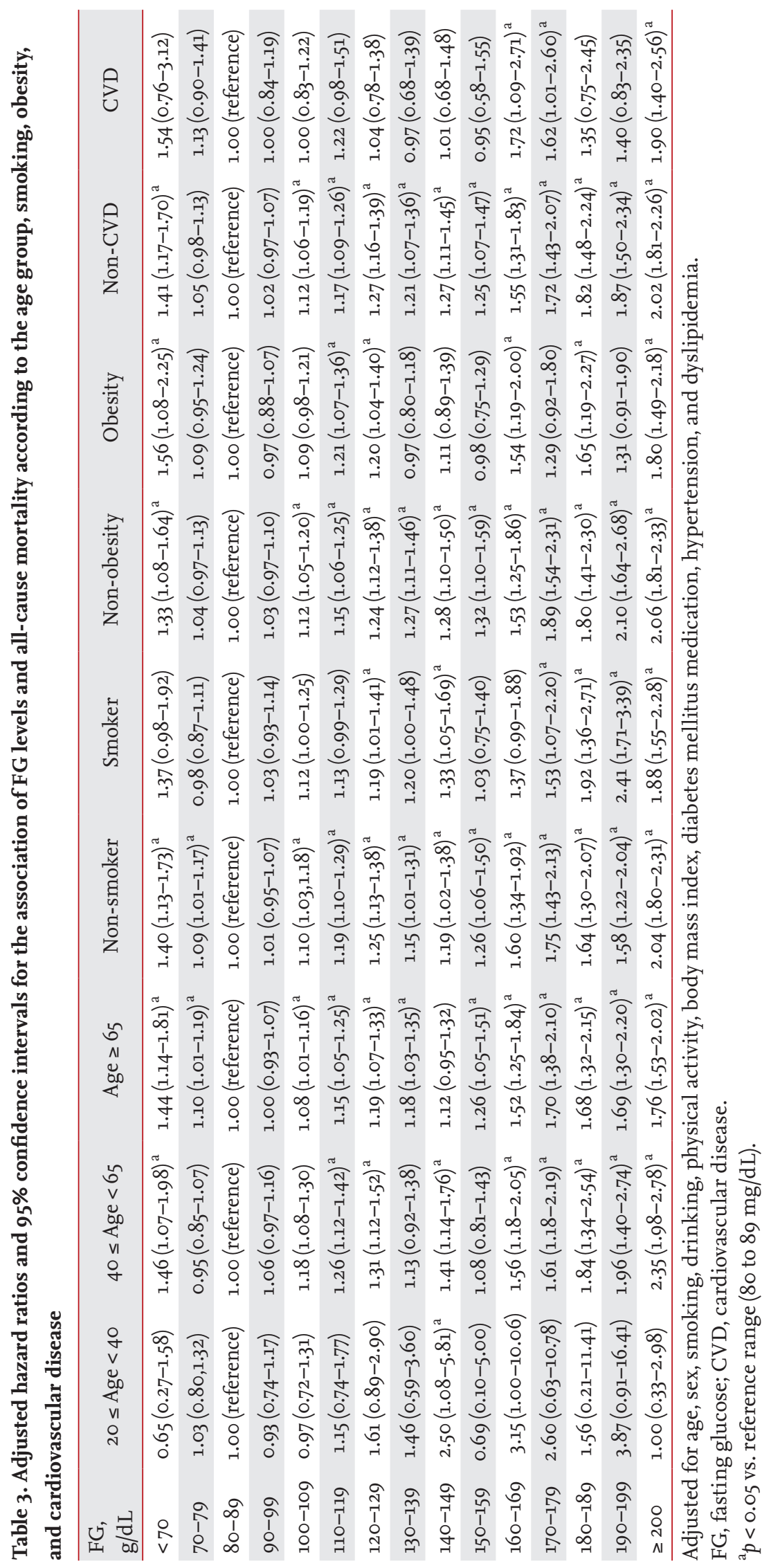


mortality reflects the consequence of hyperinsulinemia [32]. Hyperinsulinemia most often caused by insulin resistance is known to be related to colon, prostate and pancreatic cancer, which are predominantly in men [33]. Specific mortality from cancer by site has not been classified in this study, but further studies may clarify additional mechanisms of sex difference in cancer mortality, by focusing on increased insulin resistance in men with low testosterone level [34], effect of body composition and fat distribution on insulin resistance according to sex [35], and sex-dependent alteration of muscle mass in association with hyperglycemia and/or hyperinsulinemia [36]. Health care providers need to recognize this sex difference in the relationship between FG and cancer mortality risk in developing prevention and control strategies for DM.

Chronic kidney disease in patients with type $2 \mathrm{DM}$ is of particular clinical importance as it is associated with an increased risk of mortality [37,38]. However, in the general population of the present cohort study, there was no significant relationship between FG and renal disease mortality (Fig. 1). Depending on the DM medication, the association between FG level and renal mortality remained constant, except for those with FG levels < $70 \mathrm{mg} / \mathrm{dL}$ (Fig. 2). In considering the nonlinear relationship between FG and renal disease mortality, we could not exclude the possibility of misclassification, as most chronic kidney disease had been regarded as a consequence of another leading cause of death in the cohort database. Therefore, particularly in cohort studies analyzing relationships with mortality, correct information about the mortality data and cause of death is needed.

The present study had some limitations. First, while the participants in the study were from a nationally representative random sample (approximately $2.2 \%$ of the entire population with a 4.89/1,000 person-year mortality rate), a larger amount of mortality data is necessary to confirm the sex and age differences in the findings and discuss the specific causes of mortality. Second, an oral glucose tolerance test (OGTT) was not included in the dataset to completely exclude individuals with prediabetes. Studies with inclusion of various measurements (e.g., OGTT and hemoglobin A1c) will be needed for in the future. Another potential limitation of this analysis was the possibility that residual confounding factors cannot be completely excluded, though we tried to ad- just for diverse confounding variables using multivariate analyses. Nonetheless, this is the first study evaluating the association between FG and cause-specific mortality while focusing on natural deaths according to sex and age.

\section{KEY MESSAGE}

1. Low fasting glucose (FG) levels $(<70 \mathrm{mg} / \mathrm{dL})$ in individuals over age 65 and FG levels above the normal range in younger people were associated with an increased risk of all-cause and cause-specific mortality.

2. The hazard ratios for cancer mortality with FG levels $\geq 100 \mathrm{mg} / \mathrm{dL}$ were increased for men but not women.

\section{Conflict of interest}

No potential conflict of interest relevant to this article was reported.

\section{Acknowledgments}

This research was supported by a grant of the Korea Health Technology R\&D Project through the Korea Health Industry Development Institute (KHIDI), funded by the Ministry of Health and Welfare, Republic of Korea (grant number: HI18Co275). The National Sample Cohort database made by Korean National Health Insurance Service (NHIS) was used (No. NHIS-2019-2-114). The content is solely the responsibility of the authors and does not necessarily represent the official views of the NHIS.

\section{REFERENCES}

1. Rao Kondapally Seshasai S, Kaptoge S, Thompson A, et al. Diabetes mellitus, fasting glucose, and risk of cause-specific death. N Engl J Med 2011;364:829-841.

2. Ogata E, Asahi K, Yamaguchi S, et al. Low fasting plasma glucose level as a predictor of new-onset diabetes mellitus on a large cohort from a Japanese general population. Sci Rep 2018;8:13927.

3. Lee CL, Sheu WH, Lee IT, et al. Trajectories of fasting plasma glucose variability and mortality in type 2 diabe- 
tes. Diabetes Metab 2018;44:121-128.

4. Liu L, Chen X, Liu Y, et al. The association between fasting plasma glucose and all-cause and cause-specific mortality by gender: the rural Chinese cohort study. Diabetes Metab Res Rev 2019;35:е3129.

5. Kakehi E, Kotani K, Gotoh T, Kayaba K, Ishikawa S. The ratio of fasting plasma glucose to hemoglobin Alc as a predictor of all-cause mortality in individuals with normal glucose levels: the Jichi Medical School cohort study. SAGE Open Med 2019;7:2050312119860398.

6. Wang A, Liu X, Xu J, et al. Visit-to-visit variability of fasting plasma glucose and the risk of cardiovascular disease and all-cause mortality in the general population. J Am Heart Assoc 2017;6:e006757.

7. Park C, Guallar E, Linton JA, et al. Fasting glucose level and the risk of incident atherosclerotic cardiovascular diseases. Diabetes Care 2013;36:1988-1993.

8. Mongraw-Chaffin M, LaCroix AZ, Sears DD, et al. A prospective study of low fasting glucose with cardiovascular disease events and all-cause mortality: the Women's Health Initiative. Metabolism 2017;70:116-124.

9. Yi SW, Park S, Lee YH, Park HJ, Balkau B, Yi JJ. Association between fasting glucose and all-cause mortality according to sex and age: a prospective cohort study. Sci Rep 2017;7:8194.

10. Xu D, Fang H, Xu W, Yan Y, Liu Y, Yao B. Fasting plasma glucose variability and all-cause mortality among type 2 diabetes patients: a dynamic cohort study in Shanghai, China. Sci Rep 2016;6:39633.

11. Liu JJ, Lim SC, Yeoh LY, et al. Ethnic disparities in risk of cardiovascular disease, end-stage renal disease and allcause mortality: a prospective study among Asian people with type 2 diabetes. Diabet Med 2016;33:332-339.

12. Torrens JI, Skurnick J, Davidow AL, et al. Ethnic differences in insulin sensitivity and beta-cell function in premenopausal or early perimenopausal women without diabetes: the Study of Women's Health Across the Nation (SWAN). Diabetes Care 2004;27:354-361.

13. Lee JY, Ryu S, Sung KC. Association of baseline level of physical activity and its temporal changes with incident hypertension and diabetes mellitus. Eur J Prev Cardiol 2018;25:1065-1073.

14. Paffenbarger RS Jr, Hyde RT, Wing AL, Lee IM, Jung DL, Kampert JB. The association of changes in physical-activity level and other lifestyle characteristics with mortality among men. N Engl J Med 1993;328:538-545.
15. Danaei G, Finucane MM, Lu Y, et al. National, regional, and global trends in fasting plasma glucose and diabetes prevalence since 1980: systematic analysis of health examination surveys and epidemiological studies with 370 country-years and 2.7 million participants. Lancet 2011;378:31-40.

16. GBD 2016 Mortality Collaborators. Global, regional, and national under-5 mortality, adult mortality, age-specific mortality, and life expectancy, 1970-2016: a systematic analysis for the Global Burden of Disease Study 2016. Lancet 2017;390:1084-1150.

17. Wang H, Dwyer-Lindgren L, Lofgren KT, et al. Age-specific and sex-specific mortality in 187 countries, 1970-2010: a systematic analysis for the Global Burden of Disease Study 2010. Lancet 2012;380:2071-2094.

18. National Health Insurance Sharing Service. National Health Insurance Data Sharing Service [Internet]. Wonju (KR): NHISS, c2019 [cited 2020 Jun 22]. Available from: https://nhiss.nhis.or.kr/bd/ab/bdabaoooeng.do.

19. Na EJ, Lee H, Myung W, et al. Risks of completed suicide of community individuals with ICD-10 disorders across age groups: a nationwide population-based nested case-control study in South Korea. Psychiatry Investig 2019;16:314-324.

20. Lee J, Lee JS, Park SH, Shin SA, Kim K. Cohort profile: the National Health Insurance Service-National Sample Cohort (NHIS-NSC), South Korea. Int J Epidemiol 2017;46:e15.

21. American Diabetes Association. 2. Classification and diagnosis of diabetes: standards of medical care in diabetes. 2019. Diabetes Care 2019;42(Suppl 1):S13-S28.

22. Korean Standard Statistical Classification. Korean Statistical Classification of Diseases [Internet]. Daejeon (KR), c1996 [cited 2020 Jun 22]. Available from: http://kssc. kostat.go.kr/ksscNew_web/ekssc/main/main.do\#.

23. DECODE Study Group, European Diabetes Epidemiology Group. Is the current definition for diabetes relevant to mortality risk from all causes and cardiovascular and noncardiovascular diseases? Diabetes Care 2003;26:688696.

24. Wei M, Gibbons LW, Mitchell TL, Kampert JB, Stern MP, Blair SN. Low fasting plasma glucose level as a predictor of cardiovascular disease and all-cause mortality. Circulation 2000;101:2047-2052.

25. Liao HW, Saver J, Yeh HC, et al. Low fasting glucose and future risks of major adverse outcomes in people without 
baseline diabetes or cardiovascular disease: a systematic review and meta-analysis. BMJ Open 2019;9:e026010.

26. Ning F, Tuomilehto J, Pyorala K, et al. Cardiovascular disease mortality in Europeans in relation to fasting and 2-h plasma glucose levels within a normoglycemic range. Diabetes Care 2010;33:2211-2216.

27. Emerging Risk Factors Collaboration, Sarwar N, Gao P, et al. Diabetes mellitus, fasting blood glucose concentration, and risk of vascular disease: a collaborative meta-analysis of 102 prospective studies. Lancet 2010;375:2215-2222.

28. Tanne D, Koren-Morag N, Goldbourt U. Fasting plasma glucose and risk of incident ischemic stroke or transient ischemic attacks: a prospective cohort study. Stroke 2004:35:2351-2355.

29. Preiss D, Welsh P, Murray HM, et al. Fasting plasma glucose in non-diabetic participants and the risk for incident cardiovascular events, diabetes, and mortality: results from WOSCOPS 15-year follow-up. Eur Heart J 2010;31:1230-1236.

30. Selvin E, Steffes MW, Zhu H, et al. Glycated hemoglobin, diabetes, and cardiovascular risk in nondiabetic adults. $\mathrm{N}$ Engl J Med 2010;362:800-811.

31. Bjornholt JV, Erikssen G, Aaser E, et al. Fasting blood glucose: an underestimated risk factor for cardiovascular death. Results from a 22-year follow-up of healthy nondiabetic men. Diabetes Care 1999;22:45-49.
32. Jee SH, Ohrr H, Sull JW, Yun JE, Ji M, Samet JM. Fasting serum glucose level and cancer risk in Korean men and women. JAMA 2005;293:194-202.

33. Perseghin G, Calori G, Lattuada G, et al. Insulin resistance/hyperinsulinemia and cancer mortality: the Cremona study at the 15th year of follow-up. Acta Diabetol 2012;49:421-428.

34. Grossmann M. Testosterone and glucose metabolism in men: current concepts and controversies. J Endocrinol 2014;220:R37-R55.

35. Fox CS, Massaro JM, Hoffmann U, et al. Abdominal visceral and subcutaneous adipose tissue compartments: association with metabolic risk factors in the Framingham Heart Study. Circulation 2007;116:39-48.

36. Kalyani RR, Metter EJ, Ramachandran R, Chia CW, Saudek CD, Ferrucci L. Glucose and insulin measurements from the oral glucose tolerance test and relationship to muscle mass. J Gerontol A Biol Sci Med Sci 2012;67:74-81.

37. Afkarian M, Sachs MC, Kestenbaum B, et al. Kidney disease and increased mortality risk in type 2 diabetes. J Am Soc Nephrol 2013;24:302-308.

38. Davidson JA. SGLT2 inhibitors in patients with type 2 diabetes and renal disease: overview of current evidence. Postgrad Med 2019;131:251-260. 